\title{
Subsolidus solution and electrical properties of Sr-substituted bismuth magnesium niobate pyrochlores
}

\begin{abstract}
Here we investigated the structural and dielectric properties of (Bi3.36Mg0.64$\mathrm{xSrx})(\mathrm{Mg} 1.28 \mathrm{Nb} 2.72) \mathrm{O} 13.76$ (0Oे xOे.5) subsolidus solution. Sr-substituted bismuth magnesium niobate (BMSN) pyrochlores were prepared by solid-state reaction at $1025{ }^{\circ} \mathrm{C}$ over $1 \mathrm{i} 2$ days. X-ray diffraction (XRD) analysis confirmed that the BMSN pyrochlores crystallise in cubic symmetry, space group Fd3m with lattice parameters in the range 10.5968 (4)-10.5671 (17) A. The surface morphologies of these samples, as confirmed by Scanning Electron Microscopy (SEM), were composed of irregular shaped grains. Both Scherrer and Williamson-Hall methods revealed that the crystallite sizes were in the range $46 i ̈ 75 \mathrm{~nm}$. No thermal event was discernible over the temperature range $30101000{ }^{\circ} \mathrm{C}$, thus confirming the thermal stability of these materials. On the other hand, Arrhenius conductivity plots showed the BMSN pyrochlores to be highly insulating with activation energies of $\sim 1.20111 .49 \mathrm{eV}$. At $\sim 30{ }^{\circ} \mathrm{C}$ and $1 \mathrm{MHz}, \mathrm{BMSN}$ pyrochlores exhibited moderate high bulk dielectric constants, $\dot{\phi}$ $90 і ̈ 186$ and low dielectric losses, tan ŭin the order of $10 \overline{1} 2 i$ 10ī 1, respectively. Both the $\dot{\Phi}$ and $\tan$ ŭvalues of the BMSN pyrochlores showed a nearly two-fold decrease with increasing Sr concentration. Negative temperature coefficient of capacitances, TCC, ī 408 to $\overline{7} 713$ $\mathrm{ppm} /{ }^{\circ} \mathrm{C}$ were recorded over $\sim 30 \mathrm{ï} 300{ }^{\circ} \mathrm{C}$ at $1 \mathrm{MHz}$.
\end{abstract}

Keyword: Pyrochlore; Niobate compounds; Dielectric constants; Dielectric losses 